\title{
Accesibilidad al agua para consumo humano en la provincia de Salta-Argentina. Diseño de un indicador en entorno SIG
}

\section{Access to water for human consumption in the province of Salta- Argentina. Design of an indicator in the SIG context}

\author{
Emilce de las Mercedes López*, Silvia Belmonte**; María de los Ángeles García****, \\ Nilsa Sarmiento ${ }^{* * * * * * *}$ y Judith Franco ${ }^{* * * * * * * *}$
}

Citar este artículo como: López, E.; Belmonte S.; García, M. A.; Sarmiento, N. y Franco, J. (2018). Accesibilidad al agua para consumo humano en la provincia de Salta-Argentina. Diseño de un indicador en entorno SIG. Revista Nodo, 12(24), 32-45.

\section{Resumen}

El trabajo presenta un análisis espacial de la situación de la provincia de Salta en relación a la accesibilidad de la población al agua para consumo. La caracterización se realizó a partir del procesamiento de información del Censo Nacional de Población y Viviendas (INDEC, 2010). Se construyó un indicador que incorpora tres criterios de evaluación: procedencia del agua, potencialidad del acceso y tenencia de agua. Los resultados indican que un alto porcentaje de la población de Salta tiene dificultades en el acceso al agua, en particular en las zonas montañosas y el sector de Chaco salteño. La herramienta desarrollada pretende aportar al reconocimiento de la problemática del agua y a promover su abordaje territorial en la gestión nacional, provincial y local.

Palabras clave: Acceso al agua, Consumo humano, Indicador territorial, Salta.

\section{Abstract}

This paper presents a spatial analysis of the accessibility to pure water for consumption purposes. The area estudied is Salta province, located in the Northwest Argentina. Based on data from the National Population and Housing Census (2010), an indicator is defined. This indicator incorporates three evaluation criteria: origin of water, potential of access and possession of water. Results show that a high percentage of the population of Salta has difficulties in accessing water, particularly in rural areas. The developed tool aims to contribute to the acknowledgement of the water problem and to promote a territorial approach in national, provincial and local management.

Keywords: Water accessibility, Human consumption, Territorial indicator, Salta.

Fecha de recibo: 13 de octubre de 2017 • Fecha de aceptación: 25 de abril de 2018

\footnotetext{
* Instituto de Investigación en Energías No Convencionales (INENCO). Consejo Nacional de Investigaciones Científicas y Técnicas (CONICET). Correo electrónico: emigemy@yahoo.com.ar

** Instituto de Investigación en Energías No Convencionales (INENCO). Consejo Nacional de Investigaciones Científicas y Técnicas (CONICET). Correo electrónico: silvina_belmonte@yahoo.com.ar

***:* Universidad Nacional de Salta. Correo electrónico: garciamariaangeles.07@gmail.com

**** Becaria doctoral del Instituto de Investigación en Energías No Convencionales (INENCO). Consejo Nacional de Investigaciones Científicas y Técnicas (CONICET). Correo electrónico: nilsamsarmiento@gmail.com

*****: Instituto de Investigación en Energías No Convencionales (INENCO). Consejo Nacional de Investigaciones Científicas y Técnicas (CONICET). Correo electrónico: francojudita@gmail.com
} 


\section{Introducción}

En 2010, a través de la Resolución 64/292, la Asamblea General de las Naciones Unidas reconoció explícitamente el derecho humano al agua y al saneamiento, reafirmando que un agua potable limpia y el saneamiento son esenciales para la realización de todos los derechos humanos. La Resolución exhorta a los Estados y organizaciones internacionales a proporcionar recursos financieros, a propiciar la capacitación y la transferencia de tecnología para ayudar a los países, en particular a los países en vías de desarrollo, y a proporcionar un suministro de agua potable y saneamiento saludable, limpio, accesible y asequible para todos (PNUD, 2004).

Un suministro de agua es:

- Saludable: cuando está libre de microorganismos, sustancias químicas y peligros radiológicos que constituyan una amenaza para la salud humana. Las guías para la calidad del agua potable de la Organización Mundial de la Salud (OMS) proporcionan las bases para el desarrollo de estándares nacionales que, implementadas adecuadamente, garantizarán la salubridad del agua potable;

- Suficiente: cuando el abastecimiento por persona permite cubrir las necesidades de agua para beber, el saneamiento personal, la preparación de alimentos, la limpieza del hogar y la higiene personal. De acuerdo con la Organización Mundial de la Salud (OMS), son necesarios entre 50 y 100 litros de agua por persona y día para cubrir las necesidades más básicas;

- Aceptable: cuando presenta un color, olor y sabor aceptables para ambos usos, personal y doméstico. Todas las instalaciones y servicios de agua deben ser culturalmente apropiados y sensibles al género, al ciclo de la vida y a las exigencias de privacidad;

- Físicamente accesible: es prioritario garantizar servicios de agua y saneamiento accesibles físicamente dentro o situados en la inmediata cercanía del hogar, de las instituciones acadé- micas, en el lugar de trabajo o las instituciones de salud. De acuerdo con la OMS, la fuente de agua debe encontrarse a menos de 1.000 metros del hogar y el tiempo de desplazamiento para su obtención no debería superar los 30 minutos y;

- Asequible: el agua y los servicios e instalaciones de acceso al agua deben ser asequibles para todos. El Programa de las Naciones Unidas para el Desarrollo (PNUD) sugiere que el coste del agua no debería superar el 3\% de los ingresos del hogar.

De todas las características mencionadas, el foco de nuestra investigación es la accesibilidad al agua potable que generalmente está asociada a la densidad poblacional y al uso que los habitantes hacen de ella. Por lo tanto, para evaluar el derecho humano al agua en función de este criterio resulta imprescindible analizar en forma integral el contexto en que se encuentra, sus características urbanas o rurales, los aspectos socio-económicos, los bienes y servicios que presta el recurso hídrico y su componente cultural, entre otros.

\section{Situación en Argentina y en Salta}

En Argentina más de ocho millones de personas, el $20 \%$ de la población, tienen problemas para acceder al agua potable, según los datos disponibles en el Plan Nacional de Agua Potable y Saneamiento (PNAPyS) lanzado en febrero de 2016 por el Ministerio del Interior. A pesar de ese dato, no hay certezas sobre cuántos son los argentinos que efectivamente no tienen en sus casas agua confiable para beber.

En la provincia de Salta las dificultades para acceder al agua o bien la carencia del recurso hídrico son obstáculos para el desarrollo de las familias ubicadas principalmente al este de la provincia, en la zona del Chaco y al oeste de la misma en lo que se conoce como la Puna, donde las comunidades campesinas y de agricultores familiares sufren problemáticas estructurales relacionadas en muchos casos a su condición de aislamiento y pobreza, y a la marginación de las políticas públicas. 
En particular en la zona del Chaco salteño el acceso al agua se ve limitado por problemas de disponibilidad (caudal disponible), calidad (salinidad) e infraestructura (tecnologías adecuadas). En este sentido, uno de los principales problemas es la elevada concentración de substancias disueltas (mayormente sales) sobretodo de sulfato y arsénico. Estas condiciones, en la mayoría de los casos, tornan al agua en no apta para consumo humano. En particular, el problema de contaminación natural con arsénico se localiza especialmente en el abanico del río Juramento (Auge et al., 2006).

El arsénico en la llanura Chaco-Pampeana en el centro de Argentina cubre una superficie de unos 10 millones de $\mathrm{km}^{2}$. Las provincias más afectadas de la región incluyen Santiago del Estero, Chaco, Salta, Tucumán, Córdoba, Santa Fe y La Pampa (Bhattacharya P. et al. 2006). Esta gran región es una de las más densamente pobladas de Argentina, siendo la calidad del agua subterránea para el consumo humano baja, debido a las elevadas concentraciones de arsénico. En la década de los 80, Nicolli et al. (1989) señalaron que el 82\% de las muestras de agua analizadas en la región ChacoPampeana excedían los $400 \mu \mathrm{g} / \mathrm{L}$ de arsénico, superando de 8 a 40 veces los límites permisibles en agua potable en Argentina de $50 \mu \mathrm{g} / \mathrm{L}$ (Código Alimentario Argentino, 1969) y/o el valor de referencia de $10 \mu \mathrm{g} / \mathrm{L}$ que recomienda la Organización Mundial de la Salud (WHO, 1993).

En los últimos años se han desarrollado en la zona del Chaco salteño diversas estrategias institucionales en la temática del agua. Sin embargo se trata de intervenciones puntuales y el problema de acceso a este recurso vital persiste, acentuado por la situación de pobreza, marginalidad y vulnerabilidad de la población. Las alternativas de solución implementadas son variadas: desde el desplazamiento de los propios pobladores a localidades distantes en búsqueda de agua segura para el consumo o la asistencia periódica municipal con camiones cisterna, hasta la realización de pozos de agua de gran profundidad (a más de $200 \mathrm{~m}$ ). Esta última estrategia parecería ser la más eficiente para resolver de raíz el problema, sin embargo, los elevadísimos costos de inversión de esta opción tecnológica, la extensión de la zona, la densidad y distribución poblacional, dificultan una cobertura completa del área (López et al., 2013) convirtiéndola en una solución parcial.

\section{Alcance del trabajo}

En virtud de la disponibilidad de los datos publicados por el INDEC (2010), se ha realizado un estudio exploratorio de la situación en la que se encuentra el área de estudio con respecto a la provisión de agua para consumo. De este análisis, se obtuvo la información relevante para la construcción de un indicador de accesibilidad al agua en toda la provincia.

\section{Área de estudio}

El área de trabajo comprende la provincia de Salta, ubicada en el noroeste de la República Argentina, tiene una superficie de $155.488 \mathrm{Km}^{2}$ un $5,6 \%$ del territorio nacional (INDEC, 2010), limita con seis provincias y tres países (Chile, Bolivia y Paraguay). La provincia se divide en 23 departamentos y 59 municipios con una población de aproximadamente 1.214.441 habitantes (Figura 1).

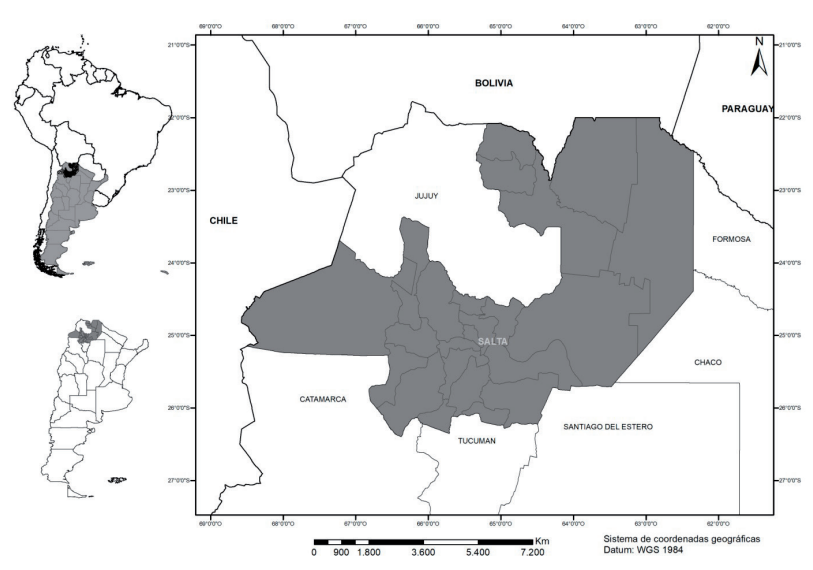

Figura 1. Área de estudio: Provincia de Salta: Capas base SIG 250. Fuente: Instituto Geográfico Nacional INDEC, 2010) 
En la provincia de Salta podemos reconocer, tomando como base las unidades del relieve, tres grandes regiones geográficas que son de oeste a este: Puna, áreas montañosas y valles intermontanos y la llanura Chaqueña (Figura 2). La llanura puneña es la forma de paisaje dominante y característica de la Puna, prolongación continental del altiplano boliviano, conformado por escalones topográficos. El régimen de precipitaciones es escaso (150 a $200 \mathrm{~mm}$ anuales), por lo que su aporte no es muy significativo, salvo en los meses de diciembre a febrero, cuando se producen las mayores precipitaciones y ocurren las crecidas de importancia. La región central de la provincia conformada por áreas montañosas y valles intermontanos incluye la Cordillera Oriental (Prepuna) y las Sierras Subandinas. La primera constituye el extremo sur de la Cordillera Oriental del Perú y Cordillera Central y Oriental de Bolivia. Esta zona presenta grandes diferencias altimétricas atravesadas por importantes cursos de agua superficial, por otro lado las posibilidades acuíferas subterráneas están dadas por los valles intermontanos alineados con rumbo meridiano, que brindan a través de pozos perforados en las llanuras aluviales importantes caudales de excelente calidad. La llanura Chaqueña constituye una gran planicie que se extiende al oriente de las Sierras Subandinas, se trata de una amplia cuenca de sedimentación donde los depósitos registran espesores de $5000 \mathrm{~m}$. La región es atravesada por las cuencas de los ríos Pilcomayo, Bermejo y Pasaje o Juramento en su sector medio. Hacia el oeste del Chaco salteño existen escasos y potentes acuíferos con material grueso, mientras que en el oriente ellos son numerosos, de poco espesor y representado por material fino, sin embargo las posibilidades de aprovechamiento son escasas debido a las limitaciones de calidad y cantidad principalmente (Paoli et al., 2012).

El trabajo plantea como objetivo analizar la criticidad del acceso al agua para consumo humano en toda la provincia, a partir de una caracterización y zonificación de esta problemática desde un enfoque territorial.

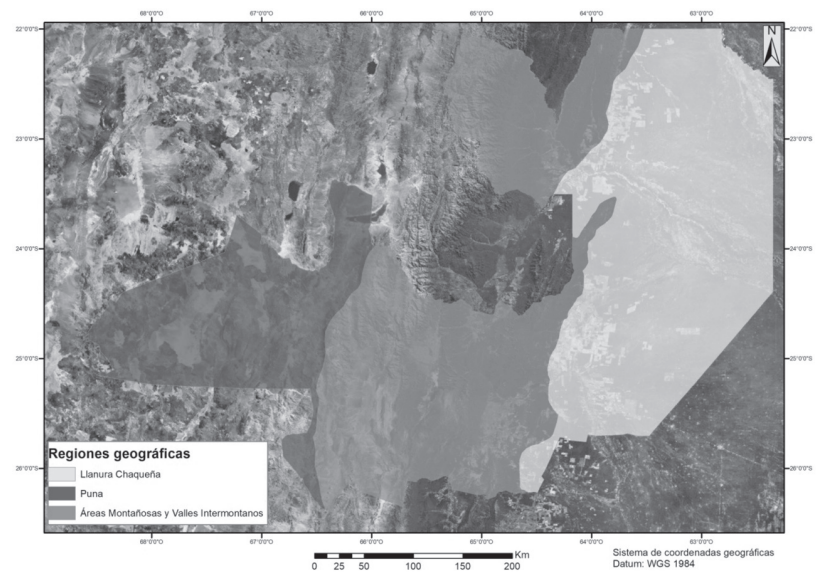

Figura 2. Regiones geográficas en la provincia de Salta. Capa base Mapa de Suelos del INTA. Fuente: Instituto de Tecnología Agropecuaria

\section{Antecedentes}

Esta investigación se desarrolla en el marco de dos proyectos realizados por el grupo Planificación Energética y Gestión Territorial del Instituto de Investigación en Energías No Convencionales (INENCO): Proyecto de Investigación Plurianual PIP CONICET N00708 (2013-2016), "Desarrollo de tecnología solar de desalinización de agua con alta producción para la mejora de condiciones de vida y sistemas productivos" y Proyecto de Investigación del Consejo de Investigación de la Universidad Nacional de Salta CIUNSA No 2019/3 (2012-2016) "Desalinización de agua para mejorar las condiciones de vida usando energía solar". A partir de estos proyectos se pretende contribuir a la mejora ambiental, social y productiva de los hábitats rurales de Salta a través del desarrollo y adecuación tecnológica de desalinizadores solares de agua. No obstante, entendiendo la complejidad del proceso de apropiación tecnológica, se planteó avanzar en el desarrollo de metodologías para la evaluación de los recursos disponibles (solar e hídrico), el análisis de la demanda social, de los factores económicos y de los aspectos ambientales. En este sentido, resultó prioritario desarrollar herramientas para identificar áreas críticas y demandas prioritarias vinculadas al acceso al agua en la región. 
Por otra parte, el uso de modelos de análisis geoespaciales se vinculó al desarrollo del Proyecto de Investigación Plurianual PIP CONICET N035 (2017-2019), "Sistema de soporte para la toma de decisiones en energías renovables (Salta - Argentina)", a partir del cual se avanzó en el trabajo con Sistemas de Información Geográfica en un entorno de software libre y con un enfoque participativo. Esto permite optimizar el acceso e integración de la información y la co-construcción de los modelos de decisión, con la finalidad de aportar de manera concreta en los procesos de planificación y gestión territorial.

Las potencialidades de la información espacio -temporal junto con técnicas algorítmicas para analizar problemas de decisión a través de estudios exploratorios basados en criterios múltiples, análisis históricos y estadísticos, permiten un análisis comparativo detallado de alternativas. La visualización geográfica permite comprender el escenario actual, proponer un escenario alternativo y predecir cambios probables con diferentes agentes, lo que es esencial para una toma de decisiones efectiva hacia el manejo sostenible de los recursos naturales (Ramachandra et al., 2017).

\section{Indicadores ambientales}

La Organización para la Cooperación y Desarrollo Económico (OCDE, 2003) define un indicador como "un parámetro o un valor derivado de parámetros, que sugiere, proporciona información acerca de, o describe el estado de un fenómeno, el medio ambiente o un área, con un significado que se extiende más allá de que estén directamente vinculados con el valor de un parámetro".

Diferentes países han desarrollado sus propios indicadores adaptados a las necesidades de cada región. En torno al agua los más conocidos son los indicadores de calidad que se pueden definir como herramientas que permiten asignar un valor de calidad al medio a partir del análisis de diferentes parámetros (Rodríguez et al., 2010). Su combinación da una visión más precisa del estado ecológico y el estado del medio biológico. Según su naturaleza existen distintos tipos de indicadores de calidad de agua como ser físico-químicos; que se basan en la combinación de parámetros físicoquímicos para proporcionar una visión global de la calidad de agua; biológicos, hidromorfológicos, entre otros.

A nivel mundial se destaca el Indicador de Calidad de Agua de la National Sanitation Foundation (ICA-NSF), desarrollado por Brown et al. (1970) para ríos de Estados Unidos y ampliamente empleado y validado o adaptado en diferentes estudios internacionales. Dinius (1987) desarrolló un ICA similar teniendo como valor agregado el planteamiento de rangos de clasificación basados en usos específicos, entre los que se destaca el del consumo humano. De manera más reciente se han planteado Indicadores de Calidad de Agua para la evaluación del recurso hídrico destinado al consumo humano, previo tratamiento, que incluyen parámetros fisicoquímicos y microbiológicos relacionados con el nivel de riesgo sanitario presente en el agua, como el Índice de Abastecimiento Público-IAP de Brasil (CETESB, 2006), el Universal Water Quality Index -UWQI (Boyacioglu, 2007), basado en Directivas de la Unión Europea, y el Drinking Water Quality Index -DWQI, de United Nations Environment Programme (UNEP, 2007). Algunos de estos indicadores pueden ser espacializados y analizados en el entorno de Sistemas de Información Geográfica (SIG).

Ramírez L. (2013) realiza un análisis estadístico con información del Censo Nacional 2010 referido a la procedencia de agua para beber y cocinar a nivel municipal en la provincia del Chaco, respecto al cumplimiento de los objetivos planteados por Naciones Unidas. A la vez establece una relación entre las necesidades actuales y los proyectos existentes para ampliar el acceso al agua por red en la provincia del Chaco. Finalmente, formula un indicador o índice de criticidad de la "procedencia de agua para beber y cocinar" considerando todas las categorías que incluye esta variable. 
Si bien existen, a nivel internacional, muchos indicadores que permiten definir la calidad del agua en función de distintos parámetros, en ocasiones estos indicadores enmascaran la realidad territorial. Es por ello que se hace necesaria la definición de indicadores locales que involucren variables representativas de las condiciones reales de acceso al agua por parte de la población. El desarrollo de un indicador que incorpore información disponible en el Censo Nacional 2010 pero que además la espacialice en el entorno de un SIG, permite ir más allá de los análisis estadísticos e identificar problemáticas importantes que muchas veces los números no reflejan.

\section{Materiales y métodos}

En la provincia de Salta, se caracterizó el acceso al agua mediante la construcción de un Indicador de accesibilidad al agua para consumo humano utilizando herramientas de análisis geoespacial y Evaluación Multicriterio (EMC).

El análisis geoespacial consiste en una integración de datos estadísticos geográficamente referenciados a través de la utilización de Sistemas de Información Geográfica (SIG). Según Haining (2003), el análisis espacial representa una colección de técnicas y modelos que explícitamente usan referencias espaciales asociadas con cada valor de una variable que está especificado dentro del sistema que se está estudiando. El análisis espacial consta de tres elementos principales: el modelo cartográfico, donde cada base de datos está representada como un mapa; los modelos matemáticos, donde los resultados dependen de la forma de interacción espacial entre los objetos en el modelo, o de relaciones espaciales, o bien de la posición geográfica de los objetos dentro del modelo; y el desarrollo y la aplicación de técnicas estadísticas para el adecuado análisis de los datos espaciales y el cual, como consecuencia, hace uso de la referencia espacial de los datos

La EMC se define como un conjunto de técnicas orientadas a asistir en procesos de decisión
(Gómez y Barredo, 2005). Se basa en la ponderación y compensación de variables que van a influir de manera positiva (aptitud) o negativa (impacto) sobre la actividad objeto de decisión o la caracterización en particular de un aspecto del territorio que requiere ser zonificado. En nuestro caso, el proceso de evaluación se orienta a obtener mapas que expresan el grado de accesibilidad al agua para consumo por parte de la comunidad. La asignación de pesos y categorías de análisis a las variables que conforman el indicador hace posible la visualización de escenarios para su estudio y comparación.

\section{Sistema de Información Geográfica Hídrico}

El equipo de trabajo cuenta con un Sistema de Información Geográfica Hídrico para la provincia de Salta que se ha convertido en una fuente de datos primarios para diversos proyectos de investigación.

Para su elaboración se recopilaron datos de diferentes fuentes. La información disponible se analizó y se organizó para facilitar su uso en el entorno del SIG. La base de datos geográfica está disponible para toda la provincia y está implementada en un sistema relacional con una arquitectura adaptada al estudio de recursos hídricos (López et al. 2013), incluyendo entre otras capas básicas: red hidrográfica, pozos de agua, infraestructura, límites político-administrativos y modelo digital de elevación. Posteriormente se incorporaron los datos de población y vivienda disponibles en el Censo Nacional 2010 (INDEC, 2010), previo procesamiento y georreferenciación. El sistema se implementó en el entorno del código QGIS, software con Licencia Libre.

\section{Indicador de accesibilidad al agua para consumo humano}

El siguiente esquema resume las principales etapas para el diseño y aplicación del indicador de accesibilidad al agua desarrollado para la provincia de Salta (Figura 3). 


\begin{tabular}{|c|c|c|c|c|}
\hline $\begin{array}{c}\text { Consulta fuentes } \\
\text { de datos }\end{array}$ & $\begin{array}{l}\text { Diseño de } \\
\text { indicador }\end{array}$ & $\begin{array}{l}\text { Procesamiento } \\
\text { capas temáticas }\end{array}$ & $\begin{array}{l}\text { Aplicación } \\
\text { del indicador }\end{array}$ & $\begin{array}{l}\text { Resultados } \\
\text { y análisis }\end{array}$ \\
\hline $\begin{array}{l}\text { - Procesamiento de } \\
\text { datos censales. } \\
\text { - Identificación } \\
\text { de variables } \\
\text { relacionadas con } \\
\text { acceso al agua. }\end{array}$ & $\begin{array}{l}\text { - Selección de } \\
\text { los criterios. } \\
\text { Ponderación } \\
\text { y planteo del } \\
\text { indicador de } \\
\text { accesibilidad. }\end{array}$ & $\begin{array}{l}\text { - Generación de capas temá- } \\
\text { ticas por cada criterio. } \\
\text { - Categorización de las } \\
\text { variables. } \\
\text { - Normalización de las capas } \\
\text { según escala de evaluación. }\end{array}$ & $\begin{array}{l}\text { Procesamiento de } \\
\text { EMC en QGIS. } \\
\text { Reclasificación } \\
\text { de las capas } \\
\text { intermedias y final. }\end{array}$ & $\begin{array}{l}\text { - Edición de mapas. } \\
\text { Aálisis espacial } \\
\text { de los resultados } \\
\text { obtenidos. }\end{array}$ \\
\hline
\end{tabular}

Figura 3. Esquema metodológico general. Fuente: elaboración propia

\section{Consulta de fuentes de datos}

La información de base fue generada a partir de datos estadísticos poblacionales (INDEC, 2010), procesados a nivel de radio censal, mínima unidad con información. Las preguntas relacionadas con el acceso al agua fueron identificadas e incorporadas al SIG para su tratamiento espacial. Los radios censales presentan tamaños variables en toda la provincia. Un sector del departamento Los Andes, en el extremo oeste de la provincia, no está habitado por lo que aparece con una trama diferencial en los mapas.

\section{Diseño del indicador de accesibilidad al agua}

Selección de criterios: Los criterios considerados para la construcción del indicador fueron definidos a partir de variables y categorías explicitadas por el Censo Nacional 2010. Se plantearon tres criterios generales para la evaluación espacial: procedencia del agua, potencialidad del acceso y tenencia de agua (Tabla 1 ).

Ponderación de los criterios: A cada una de estas variables se le asignó un peso de acuerdo a su importancia para garantizar el acceso a agua segura para las personas. La procedencia del agua resultó el criterio más importante (peso 10), seguido por la distribución espacial de la población (peso 8) que determina la potencialidad de "acercar el agua" por distintos medios a las comunidades. Finalmente, la tenencia del agua resultó una variable menos significativa (peso 4) porque implica que la pobla- ción ya tiene acceso al agua y lo que define es la distancia que debe recorrer para su obtención.

Fórmula: El Indicador de Accesibilidad al Agua (IAA) para consumo humano construido se expresa con la siguiente notación aritmética:

$$
\mathrm{IAA}=\mathrm{P}^{*} \mathrm{p}_{1}+\mathrm{PA}^{*} \mathrm{p}_{2}+\mathrm{T}^{*} \mathrm{p}_{3}
$$

Donde

$$
\begin{aligned}
& \mathrm{p}_{1-3}=\text { peso de los criterios } \\
& \mathrm{P}=\text { Procedencia del agua } \\
& \mathrm{PA}=\text { Potencialidad de acceso } \\
& \mathrm{T}=\text { Tenencia del agua }
\end{aligned}
$$

Un valor bajo obtenido para el indicador refiere a un mayor nivel de criticidad en el acceso al agua por la población.

\section{Generación de capas temáticas}

Las variables consideradas en la construcción del Indicador de Accesibilidad al Agua fueron representadas como mapas temáticos utilizando el radio censal como mínima unidad de análisis y aplicando un gradiente de color para la representación espacial. Las capas temáticas de cada criterio se obtuvieron mediante una construcción estadística que tiene como origen los datos correspondientes a todas las categorías (atributos) de cada variable por radio censal. El valor asignado surge de la suma ponderada de las viviendas correspondientes a cada atributo dentro del radio censal, dividido el total de viviendas (ver categorías y valores en Tabla 1 ). 
Tabla 1. Planteo de criterios y categorías para la evaluación de la accesibilidad al agua para consumo humano

\begin{tabular}{|c|c|c|c|c|c|}
\hline Criterios & Peso & Categorías & Descripción & Calificación & Valor \\
\hline \multirow{6}{*}{$\begin{array}{l}\text { Procedencia del agua } \\
\text { Refiere a la fuente } \\
\text { y el sistema de } \\
\text { abastecimiento del agua } \\
\text { que se utiliza en el hogar } \\
\text { para beber y cocinar. } \\
\text { En caso de abastecerse } \\
\text { con más de una fuente, } \\
\text { se considera la fuente } \\
\text { que predomina en el uso } \\
\text { cotidiano del hogar. }\end{array}$} & \multirow{6}{*}{10} & Red pública & $\begin{array}{l}\text { Sistema de captación, tratamiento y } \\
\text { distribución de agua mediante una red de } \\
\text { tuberías comunal sometida a inspección } \\
\text { y control por las autoridades públicas. El } \\
\text { sistema puede estar a cargo de un organismo } \\
\text { público, cooperativa o empresa privada. }\end{array}$ & Óptima & 10 \\
\hline & & $\begin{array}{l}\text { Perforación con } \\
\text { bomba a motor }\end{array}$ & $\begin{array}{l}\text { Sistema de captación que consiste en la } \\
\text { extracción del agua de las napas profundas, } \\
\text { a través de un medio mecánico de elevación } \\
\text { con motor. }\end{array}$ & Muy buena & 8 \\
\hline & & $\begin{array}{l}\text { Perforación con } \\
\text { bomba manual }\end{array}$ & $\begin{array}{l}\text { Sistema de captación que consiste en la } \\
\text { extracción del agua de las napas profundas, } \\
\text { a través de un medio mecánico de elevación } \\
\text { manual. }\end{array}$ & Buena & 7 \\
\hline & & Pozo & $\begin{array}{l}\text { Sistema de captación que consiste en la } \\
\text { extracción del agua de las napas superficiales } \\
\text { a través de un medio mecánico de elevación } \\
\text { (que puede ser manual o a motor) o bien } \\
\text { a través de un balde o similar (sin utilizar } \\
\text { medios mecánicos de elevación). }\end{array}$ & Satisfactoria & 5 \\
\hline & & $\begin{array}{l}\text { Transporte por } \\
\text { cisterna }\end{array}$ & $\begin{array}{l}\text { Provisión por medio de un camión tanque, un } \\
\text { tren aguatero, etc. }\end{array}$ & Deficiente & 3 \\
\hline & & $\begin{array}{l}\text { Otras fuentes de } \\
\text { abastecimiento }\end{array}$ & $\begin{array}{l}\text { Abastecimiento de agua de lluvia, de ríos, } \\
\text { canales, arroyos o acequia. }\end{array}$ & Crítica & 1 \\
\hline $\begin{array}{l}\text { Potencialidad } \\
\text { del acceso } \\
\text { Representa la }\end{array}$ & \multirow{3}{*}{8} & Urbana & Población mayor o igual a 2000 habitantes. & Alta & 9 \\
\hline $\begin{array}{l}\text { a infraestructura de } \\
\text { distribución espacial de } \\
\text { la población. Considera }\end{array}$ & & Rural agrupada & Población menor a 2000 habitantes. & Moderada & 6 \\
\hline $\begin{array}{l}\text { que a mayor agrupación } \\
\text { poblacional existe mayor } \\
\text { potencial de acceso al } \\
\text { corto plazo. }\end{array}$ & & Rural dispersa & Población dispersa. & Baja & 3 \\
\hline \multirow{3}{*}{$\begin{array}{l}\text { Tenencia de agua } \\
\text { Está relacionado con la } \\
\text { forma de acceder al agua } \\
\text { en la vivienda }\end{array}$} & \multirow{3}{*}{4} & $\begin{array}{l}\text { Por cañería } \\
\text { dentro de la } \\
\text { vivienda }\end{array}$ & $\begin{array}{l}\text { Sistema de suministro de agua conectado a } \\
\text { una red de tuberías por medio de la cual se } \\
\text { distribuye el agua en su interior. }\end{array}$ & Óptima & 10 \\
\hline & & $\begin{array}{l}\text { Fuera de la } \\
\text { vivienda pero } \\
\text { dentro del } \\
\text { terreno }\end{array}$ & $\begin{array}{l}\text { Sistema de suministro de agua dentro de } \\
\text { los límites que ocupa el terreno pero que no } \\
\text { dispone de una red de tuberías para distri- } \\
\text { buirla hacia el interior de la vivienda. }\end{array}$ & Buena & 7 \\
\hline & & Fuera del terreno & $\begin{array}{l}\text { Sistema de suministro de agua que se } \\
\text { encuentra fuera de la vivienda y fuera de los } \\
\text { límites que ocupa el terreno. }\end{array}$ & Deficiente & 3 \\
\hline
\end{tabular}


Valor asignado a cada radio censal:

$$
\mathrm{P} / \mathrm{PA} / \mathrm{T}=\sum \mathrm{r}_{\mathrm{i}^{*}} \mathrm{~V}_{\mathrm{i}} / \mathrm{N}
$$

Donde

$$
\begin{aligned}
\mathrm{r}_{\mathrm{i}}= & \text { frecuencia de hogares por } \mathrm{i} \text { atributo, con } \mathrm{i} \\
& \text { que varía de } 1 \text { a n, de acuerdo a la variable } \\
& \text { considerada. } \\
\mathrm{N}= & \text { Total de hogares } \\
\mathrm{i}= & \text { atributo correspondiente }
\end{aligned}
$$

\section{Resultados y análisis}

Los resultados obtenidos se representaron espacialmente en tres mapas temáticos (uno para cada variable/criterio) y un mapa final correspondiente a la distribución del indicador IAA (Figuras 4 a 7). Por otra parte, se realizó un análisis comparativo de los resultados espaciales versus los datos estadísticos generales del censo para la provincia (Tabla 2) considerando las mismas categorías planteadas para la aplicación espacial del IAA. Los resultados de la zonificación fueron presentados para su discusión en talleres participativos interinstitucionales.

Una primera observación de la Tabla 2 indicaría que altos porcentajes de la población de la provincia cuenta con buena accesibilidad física al agua. De acuerdo a los resultados del último censo, Salta tiene porcentajes elevados respecto a la variable Procedencia de agua, donde el 91\% de los hogares está conectado a la red pública. De la misma manera, la potencialidad del acceso resulta alta, ya que el $87 \%$ de la población se encuentra en zonas urbanas. Asimismo, el 94\% de los hogares cuenta con acceso al agua dentro de la vivienda. Este análisis a nivel provincial podría llevar a presuponer que la población de

\begin{tabular}{|c|c|c|c|}
\hline \multirow{2}{*}{ Criterios } & \multirow{2}{*}{ Categorías } & \multicolumn{2}{|c|}{ Totales } \\
\hline & & Hogares & $\%$ \\
\hline \multirow{7}{*}{$\begin{array}{l}\text { Procedencia del agua para beber y } \\
\text { cocinar }\end{array}$} & Red pública & 273.466 & 91 \\
\hline & Perforación con bomba a motor & 8.387 & 3 \\
\hline & Perforación con bomba manual & 913 & 0.3 \\
\hline & Pozo & 3.735 & 1 \\
\hline & Transporte por cisterna & 5.951 & 2 \\
\hline & Agua de lluvia, río, canal, arroyo o acequia & 7.342 & 2 \\
\hline & Total & 299.794 & 100 \\
\hline \multirow{5}{*}{ Potencialidad del acceso } & & Población & $\%$ \\
\hline & Urbana & 1.057 .951 & 87 \\
\hline & Rural Agrupada & 59.104 & 5 \\
\hline & Rural Dispersa & 97.386 & 8 \\
\hline & Total & 1.214 .441 & 100 \\
\hline \multirow{5}{*}{ Tenencia de agua } & & Hogares & $\%$ \\
\hline & Por cañería dentro de la vivienda & 229.157 & 76 \\
\hline & Fuera de la vivienda pero dentro del terreno & 53.198 & 18 \\
\hline & Fuera del terreno & 17.439 & 6 \\
\hline & Total & 299.794 & 100 \\
\hline
\end{tabular}
Salta no tiene un problema real de accesibilidad al agua para consumo. No obstante, los valores así presentados enmascaran una realidad diferente que se visualiza al realizar un análisis a

Tabla 2. Resultados estadísticos publicados por INDEC (2010) para el total provincial 
nivel espacial. Las condiciones de vulnerabilidad en el territorio, en particular de las extensas áreas rurales de Salta, no se evidencian al considerar los totales provinciales.

En el caso del mapa "Procedencia de agua" (Figura 4 ), se observa que aproximadamente el $50 \%$ de la superficie total de la provincia se encuentra en una situación deficiente a crítica en relación a la fuente de abastecimiento de agua para consumo. Esta situación implica una predominancia en la procedencia de agua de pozo superficial, camión cisterna, agua de lluvia, río, canal, arroyo o acequia, situación común en la mayoría de las zonas rurales de la provincia. En general, sólo los radios censales que incluyen sectores urbanos y algunos sectores rurales bien consolidados presentan una buena condición de procedencia de agua con acceso por red pública o perforaciones de agua con bomba. Considerando la superficie de Salta con predominancia de acceso al agua por red, lo que implica sistemas implementados por organismos públicos, cooperativas o empresas y controlados por autoridades públicas, sólo se cubre el 7,5\% del área poblada provincial.

El análisis de la "Potencialidad del acceso" (Figura 5) indica que el $89 \%$ de la superficie de la provincia presenta población rural dispersa, lo que dificulta el potencial desarrollo y mejora de infraestructuras apropiadas. Por el contrario, el $87 \%$ de la población se encuentra concentrada en los grandes centros urbanos, que espacialmente sólo representan el 3\% de la superficie poblada provincial con alta viabilidad de implementar mejoras estructurales al corto plazo.

La zonificación del criterio "Tenencia del agua" (Figura 6) muestra que sólo en el 15\% de la superficie provincial cuenta con un sistema de provisión de agua por cañería dentro de la vivienda, mientras que en el $85 \%$ restante el suministro de agua queda por fuera del hogar (quedando incluso fuera del terreno de la vivienda en un $10 \%$ de la superficie analizada total).

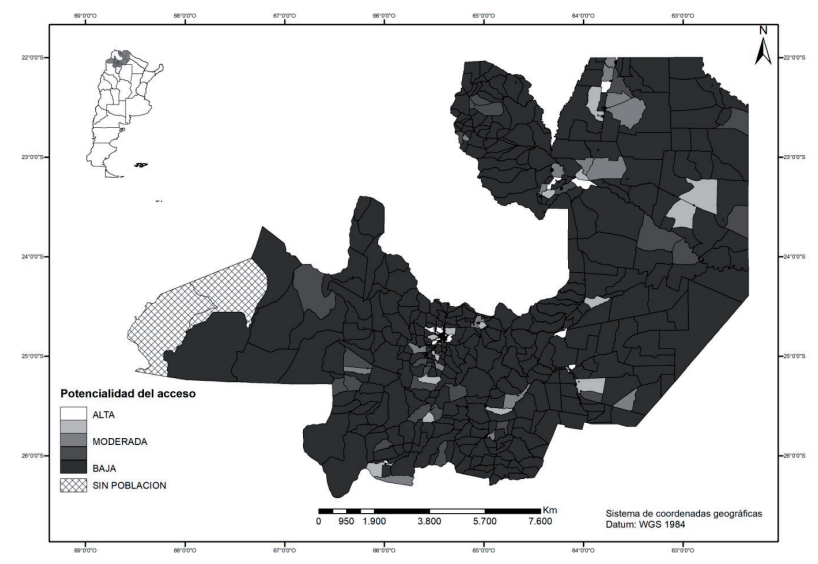

Figura 4. Zonificación de la variable Procedencia del agua. Fuente: elaboración propia

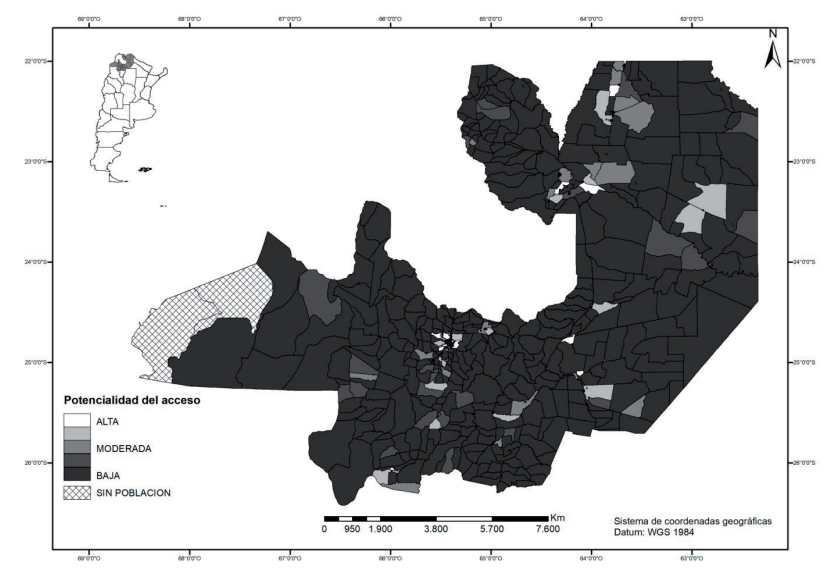

Figura 5. Zonificación de la variable Potencialidad de acceso. Fuente: elaboración propia

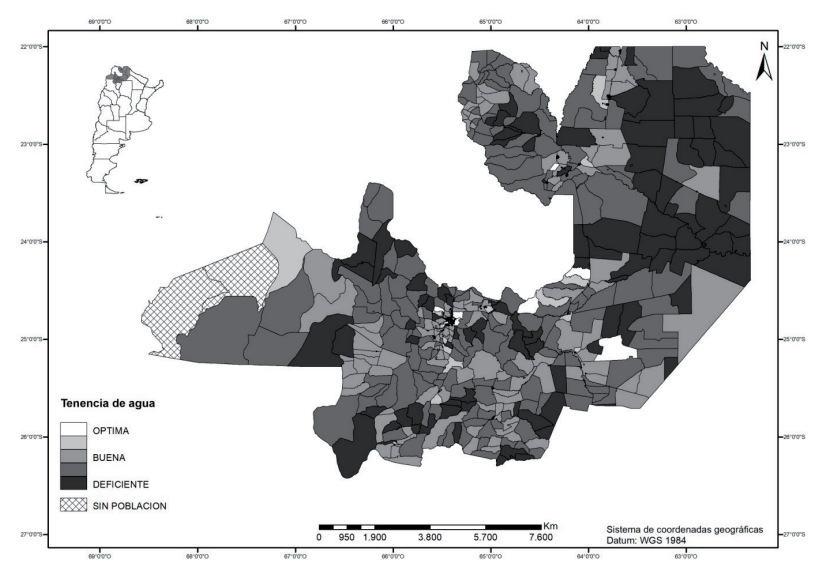

Figura 6. Zonificación de la variable Tenencia del agua. Fuente: elaboración propia 


\section{Aplicación del Indicador de accesibilidad al agua para consumo humano}

Las capas temáticas generadas fueron combinadas en el SIG mediante la aplicación de la fórmula para el cálculo del Indicador. Éste puede tomar valores en un rango de 46 a 212, donde a mayor valor del indicador, mejor situación de la población en relación a que el recurso hídrico para consumo es físicamente accesible. Si se analiza desde la criticidad del acceso, un menor valor del indicador muestra una situación más crítica de accesibilidad al recurso.

Los valores obtenidos para el IAA fueron clasificados mediante el método de Rupturas naturales (Jenks, 1967) que se basan en las agrupaciones naturales inherentes a los datos. Los cortes de clase se caracterizan porque agrupan mejor los valores similares y maximizan las diferencias entre clases. Los límites de las entidades quedan establecidos dónde hay diferencias considerables entre los valores de los datos, en función de los cuales se han establecido las siguientes condiciones de accesibilidad: buena, moderada, baja y crítica.

El mapa final de "Accesibilidad al agua para consumo" (Figura 7), resultado de la combinación de las variables anteriormente presentadas, revela que en más del $95 \%$ de la superficie de la provincia el acceso al

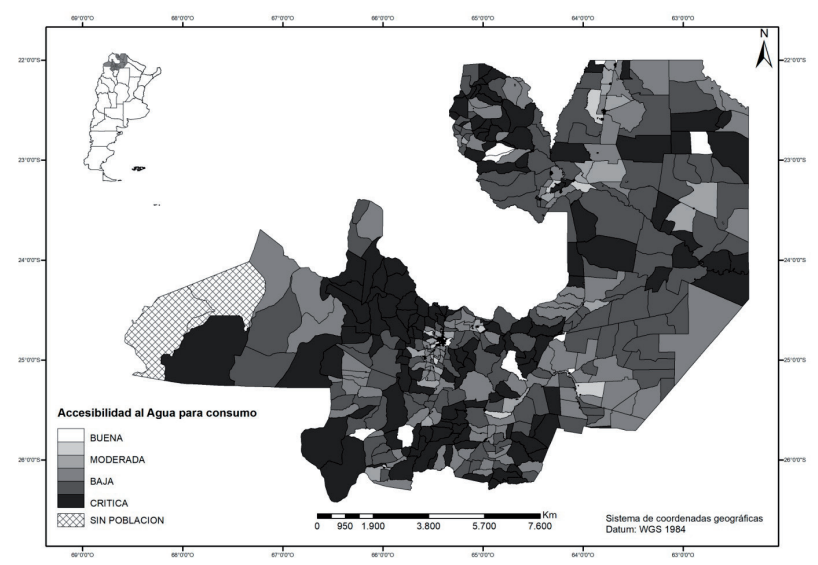

Figura 7. Zonificación provincial del Indicador de Accesibilidad al Agua. Fuente: elaboración propia. agua resulta conflictivo ( $72 \%$ accesibilidad crítica y $23 \%$ accesibilidad baja). La distribución espacial de esta problemática alcanza toda la provincia pero se concentra especialmente en la Puna, los sectores montañosos y el Chaco salteño. Sólo en un 5\% de la superficie provincial el indicador IIA adopta valores de moderados a buenos.

\section{Conclusiones y reflexiones finales}

Los resultados obtenidos en el mapeo del IIA resaltan una fuerte problemática de accesibilidad al agua en la provincia de Salta, Noroeste de Argentina. Si bien el número de familias afectadas sólo representa un $10 \%$ de la población provincial, las condiciones de desigualdad son extremas y resultan críticas para las extensas áreas rurales. En estas zonas, no sólo no existe actualmente acceso al recurso agua de manera segura, sino que además no tiene perspectivas de ser solucionado al corto plazo por complejas condiciones de aislamiento y vulnerabilidad.

El análisis realizado se traduce en un llamado de atención para el planteo de políticas públicas de acceso al agua más inclusivas que consideren la diversidad de condiciones geográficas y demográficas locales. Los análisis estadísticos pueden resultar insuficientes para analizar problemáticas de esta índole, que afectan principalmente los sectores rurales, cuando la mayoría de la población se concentra en zonas urbanas. Una mirada integral y atenta de la provincia permite identificar un problema "extenso" en el sentido de superficie afectada, si bien porcentualmente no pareciera ser una problemática profunda.

La costumbre tendenciosa de generar proyectos y programas que atiendan el mayor número de beneficiarios con respuestas tecnológicas unificadas y uniformes para todos, juega en detrimento de estas comunidades aisladas y dispersas que requieren soluciones diversas y creativas para resolver un derecho fundamental de las personas: el derecho al agua. En las palabras de Eduardo Bertea "El agua es un derecho incuestionable y universal. Esto debe 
difundirse y el Estado tiene el deber de asegurar que se cumpla" (PEyGeT, 2016).

Múltiples iniciativas de acción colectiva y lideradas por diversas instituciones, se vienen desarrollando desde hace años para mejorar el acceso y distribución de agua en comunidades rurales del país y en particular en el chaco salteño (INTA, 2009; Belmonte et al., 2016; García et al., 2017). No obstante, la problemática persiste. Sin negar la centralidad y responsabilidad que le compete al Estado a la hora de afrontar las soluciones, el Instituto Nacional de Tecnología Agropecuaria (INTA, 2009) en su publicación "Acceder al agua" propone buscar la clave en la articulación de saberes, la complementariedad de capacidades y la construcción conjunta de soluciones.

Lamentablemente, documentos actuales de planificación nacional sobre Agua y Saneamiento (Dirección Nacional de Agua Potabley Saneamiento, 2017) desconocen estas lecciones aprendidas, y la realidad de las zonas rurales quedan invisibilizadas en análisis cuantitativos y propuestas económica y socialmente rentables pero no inclusivas de la diversidad de nuestra región, lo que contrasta fuertemente con el lema del plan "Cobertura universal y sostenibilidad de los servicios".

La herramienta desarrollada a partir de este trabajo pretende aportar al reconocimiento de la problemática compleja del agua, promoviendo un abordaje territorial para la búsqueda de soluciones integradas en su gestión nacional, provincial y local. Reconociendo el alcance puntual y limitado de este aporte "científico-técnico", se espera poner a disposición la información generada en espacios participativos de reflexión y gestión (tarea que ya se viene realizando en talleres interinstitucionales y mesas de agua) y plataformas web de consulta abierta en colaboración con organismos públicos provinciales (Secretaría de Ambiente y Desarrollo Sustentable y programa IDESA - Infraestructura de Datos Espaciales de Salta-). Es la intención sumar este aporte y el compromiso del grupo de investigación al enorme desafío que representa mejorar la accesibilidad al agua segura en la provincia de Salta.

\section{Referencias bibliográficas}

Auge, M., Wetten, C., Baudino, G., Bonorino, G., Gianni, R., González, N., Grizinik M., Hernández, M., Rodríguez, J., Sisul, A., Tineo, A. \& Torres, C. (2006). Hidrogeología de Argentina. Boletín Geológico y Minero, 117 (1), pp. 7-23.

Bhattacharya, P., Claesson, M., Bundschuh, J., Sracek, O., Fagerberg, J., Jacks, G. et al. (2006). Distribution and mobility of arsenic in the Rio Dulce alluvial aquifers in Santiago del Estero Province, Argentina. Sci Total Environ; 358, pp. 97-120.

Belmonte, S., López, E., Sarmiento, N., García, M. A., Caso, R., Goareguer, A. \& Franco, J. (2016). Acceso al agua en el Chaco salteño: Mirada integral a un problema no resuelto. Espacio agua para el Desarrollo. $3^{\circ}$ Encuentro Mundial Gran Chaco, Villa Montes, Bolivia.

Boyacioglu, H. (2007). Development of a water quality index based on a European classification scheme. Water SA, Vol. 33, No. 1, pp. 101-106.

Brown, R., Macclelland, N., Deininger, R. \& Tozer, R. (1970). A Water Quality Index - Do We Dare? Water and Sewage Works, Vol. 11, pp. 339-343.

Código Alimentario Argentino. Capítulo XII -Bebidas hídricas, agua y agua gasificada. Agua potable Artículo 982 - Resolución Conjunta SPRyRS y SAGPyA Nº 68/2007 y N 196/2007. Recuperado de: www.anmat.gov.ar/alimentos/ codigoa/Capitulo_XII.pdf 12/09/13

Companhia de Tecnologia de Saneamento Ambiental -CETESB (2006). Relatório de qualidade das águas interiores no estado de São Paulo. Anexo V. Brasil: CETESB.

Dirección Nacional de Agua Potabley Saneamiento. Subsecretaría de Recursos Hídricos. Ministerio del Interior (2017). Plan Nacional de Agua Potable y Saneamiento. Cobertura Universal y Sostenibilidad de los Servicios. Lineamientos y Principales acciones. Segunda versión, junio de 2017. Recuperado de: https://www.argentina. 
gob.ar/sites/default/files/interior_agua_plan_ agua_saneamiento.pdf

Dinius, S. H. (1987). Design of an Index of Water Quality. Water Resources Bulletin, Vol. 23, No. 11, pp. 833-843.

García, M. et al. (2017). La gestión inter-institucional como tecnología de acceso al agua en el Chaco Salteño. XXVI Congreso Nacional del Agua. Córdoba, Argentina: CONAGUA.

Gómez, M. \& Barredo, J. (2005). Sistemas de Información Geográfica y Evaluación Multicriterio en la Ordenación del Territorio. Madrid: Ra-Ma.

Haining, R.P. (2003). Spatial data analysis: Theory and practice. Cambridge: Cambridge University Press.

INDEC (2015). Unidades Geoestadísticas. Cartografía y códigos geográficos del Sistema Estadístico Nacional. Buenos Aires: INDEC.

INDEC (Instituto Nacional de Estadística y Censos) (2010). Censo Nacional de Población y Vivienda. Base de Datos Redatam.

Instituto Nacional de Tecnología Agropecuaria (INTA). Sistema de Información Geográfica. Recuperado de: http://inta.gob.ar/servicios/ sistema-de-informacion-geografica.

Instituto Nacional de Tecnología Agropecuaria (2009). Acceder al agua: relatos y reflexiones desde algunas experiencias de organización en los territorios. Compiladores Miguel Barreda, Sandra Ledesma. Buenos Aires: INTA.

Jenks, G. F. (1967). The Data Model Concept in Statistical Mapping. International Yearbook of Cartography $\mathrm{n}^{\circ}$ 7: pp. 186-190.

López, E., Belmonte, S.\& Franco J. (2013). Elaboración de un Sistema de Información Geográfica hídrico para evaluar potenciales aplicaciones de energía solar en el Chaco Salteño. Revista AVERMA (Avances en Energías Renovables y Medio Ambiente). Vol. 17, pp 43-53.

Nicolli, H.B., Suriano, J.M., Gómez Peral, M.A., Ferpozzi, L.H. \& Baleani, O.M. (1989). Ground- water contamination with arsenic and other trace elements in an area of the Pampa, Province of Córdoba, Argentina. Environ Geol Water Sci 14(1), pp. 3-16.

Paoli, H., Volante, J., Noe, Y., Vale, L., Castrillo, S., Osinaga, R., Chafatinos, T. \& Nadir, A. (2012). Adecuación a un sistema de información geográfica del estudio "Los Suelos del NOA (Salta y Jujuy), Nadir, A. \& Chafatinos, T., 1990". Argentina: INTA. Recuperado de: https://inta.gob.ar/ sites/default/files/script-tmp-regiones_geo.pdf

Planificación Energética y Gestión Territorial - Instituto de Investigaciones en Energía No Convencional (INENCO - CONICET - UNSa) (2016). Taller Interinstitucional: "Gestión del Agua en el Chaco Salteño". Informe del taller. Colaboradores en la organización: Secretaría de Recursos Hídricos de la Provincia de Salta e Instituto Nacional de Tecnología Industrial Centro Salta (INTI). Salta: Biblioteca Provincial.

PNUD (2004). Water as a Human Right? IUCN Environmental Policy and Law Paper N51. Recuperado de: https://portals.iucn.org/library/ sites/library/files/documents/EPLP-051.pdf

Ramachandra T.V.; Tara N.M. Bharath Setturu (2017). Web Based Spatial Decision Support System for Sustenance of Western Ghats Biodiversity, Ecology and Hydrology. Creativity and Congition in Art and Design, Edited by Aneesha Sharma and Jamuna Rajeswaran, pp. 58-70. Recuperado de: http://wgbis.ces.iisc.ernet.in/ energy/water/paper/SDSS/index.html

RamírezL.(2013).ElaccesoalaguapotableenelChaco [Argentina] y los progresos hacia el objetivo del milenio. Una mirada a través de la elaboración de un índice de criticidad. Revista Geográfica Digital. IGUNNE. Facultad de Humanidades. UNNE. Año 10. No 20. Chaco: Resistencia. Recuperado de: http://hum.unne.edu.ar/revistas/geoweb/de fault.htm

Resolución A/RES/64/292 (2010). Asamblea General de las Naciones Unidas. Recuperado 
de: http://www.un.org/ga/search/view_doc.asp? symbol=A/RES/64/292\&Lang=S

Rodríguez, A., Reolón, L. \& Pertusi, L. (2010). Calidad de las aguas. Herramientas y experiencias de proyectos aplicados (índice e indicadores de calidad de agua), Módulo 4, Edición 5. Argentina: CEDDET.

United Nations Environment Programme -UNEP (2007). Global Drinking Water Quality Index Development and Sensitivity Analysis Report. Ontario, Canadá: UNEP.
Watkins, K., Ross-Larson, B., De Coquereau-mont, M. \& Trott, C. (2006). Informe sobre desarrollo humano 2006. Más allá de la escasez: Poder, pobreza y la crisis mundial del agua. New York: PNUD.

World Health Organization (1993). Guidelines for drinking-water quality. 2nd ed. Geneva, Switzerland: WHO. Vol. 1. Recuperado de: www. who.int/watersanitation_health/dwq/gdwq2v1/ en/ 